\title{
Visual - Force Control and Structured Light Fusion to Improve Recognition of Discontinuities in Surfaces
}

\author{
J. Pomares, P. Gil, G. J. García, F. Torres \\ Department of Physics, System Engineering and Signal Theory \\ University of Alicante \\ PO Box 99, 03080 Alicante \\ Spain \\ \{jpomares, Pablo.Gil,.gjgg, Fernando.Torres\}@ua.es
}

\begin{abstract}
This paper describes a method to detect changes in given surfaces tracked by the robot end-effector. To do so, first an approach to combine visual and force information is described. By filtering the interaction forces, a parameter which provides the probability of a change in the tracking surface is obtained. In order to obtain a more accurate determination of the change in the surface, information from a laser located at the robot end-effector is also employed. Therefore, a robust method for detecting surface discontinuities which employs force and visual information jointly with structured light is presented.
\end{abstract}

\section{INTRODUCTION}

Nowadays there are a lot of industrial applications in which is necessary to combine visual and force information during the tracking of surfaces. Within these applications we can mention polishing, deburring, machining or assembly. Several approaches have been proposed for the interaction control between the robot and the workspace; these approaches are based on hybrid control, impedance control or parallel control. A review of these approaches is detailed in [5]. So far, most of the applications developed to combine visual and force information employ hybrid control, such as studies like [1] which extend the "task frame" formalism [3]. In hybrid approaches, the workspace must be precisely known, and a division of the directions controlled by force and others controlled by position (i.e., using a visual servoing system) are first carried out. When a change in the surface occurs, the previously mentioned directions must be modified. Therefore, it is required to recognize the contact surface so that if this surface has a discontinuity a new recognition of the surface must be developed at the moment in which the surface changes. In this paper, we are not interested in developing a surface recognition method (In order to do so it is supposed that our previous algorithms [15] have been employed). However, this paper describes a robust method to detect changes in the contact surface employing a multisensorial system composed of an eye-in-hand camera system, a force sensor and a laser.

Now, the visual servoing systems are a well known approach to guide a robot by using image information obtained from an object observed by an eye-in-hand camera system [10]. In applications in which it is required the interaction between the robot and a given surface, the image information must be combined with that obtained from other sensors in order to improve the system behaviour. Furthermore, additional sensorial information can be integrated in the visual loop, like the one obtained from a laser. Attaching a structured light emitter to the camera makes it possible to project visual marks onto the object surface which can be used in the control loop for positioning the camera located at the end-effector. In [13] a structured light and image-based visual servoing method for plane-to-plane positioning is proposed. In [11] other studies in which jointly use visual and structured light information are described. In this paper, a visual servoing approach is defined to track trajectories and to detect surface changes. Force and structured light information are included in the visual loop.

This paper is organized as follows: The visual-force control system to track surfaces is first described in Section II. Section III shows a method based on the Generalized Likelihood Ratio to detect surface discontinuities. In Section IV, a strategy to determine surface discontinuities in the image space based on structured light is presented. Section V describes the joint use of the two previous methods in order to obtain a robust estimation of the surface discontinuity. In Section VI, experimental results, using an eye-in-hand camera system, confirm the validity of the proposed algorithms. The final section presents the main conclusions obtained.

\section{VISUAL-FORCE CONTROL TO TRACK SURFACES}

Now, we consider the task of tracking a surface using visual and force information. The visual loop carries out the tracking of the desired trajectory in the image space. In order to do so an image-based control scheme is used [12]. This control scheme regulates to 0 the vision-based task function represented in (1):

$$
\boldsymbol{e}=\hat{\mathbf{J}}_{\mathrm{f}}^{+} \cdot\left(\mathbf{s}-\mathbf{s}_{\mathrm{d}}(t)\right)
$$

where:

- $\quad \mathbf{s}=\left[\boldsymbol{f}_{1}, \boldsymbol{f}_{2}, \ldots, \boldsymbol{f}_{\mathrm{M}}\right]^{\mathrm{T}}$ are the visual features extracted from the image.

- $\hat{\mathbf{J}}_{\mathrm{f}}^{+}$is the estimation of the pseudoinverse of the interaction matrix [10]. 
- $\mathbf{s}_{\mathrm{d}}$ is the desired trajectory in the image. Therefore $\mathbf{s}_{\mathrm{d}}(t)$, is the value of the desired trajectory at time $\mathrm{t}$.

- $\gamma(\mathrm{t})$ will be considered as the desired robot 3-D trajectory that will be tracked (i.e., the desired 3-D trajectory of the camera at the end-effector) For this study, we have employed planners already described in our previous studies [14].

To carry out the tracking of the trajectory, the following velocity must be applied to the robot (with respect to the coordinate frame located at the eye-in-hand camera):

$$
\boldsymbol{v}_{\mathrm{V}}^{\mathrm{C}}=-\lambda_{\mathrm{V}} \cdot \boldsymbol{e}+\widehat{\mathbf{J}}_{\mathrm{f}}^{+} \cdot \frac{\partial \mathbf{s}_{\mathrm{d}}(t)}{\partial t}
$$

where $\lambda_{\mathrm{V}}>0$ is the gain of the proportional controller.

Considering $\boldsymbol{F}$ and $\boldsymbol{F}_{\mathrm{d}}$ the obtained and desired interaction forces; $\lambda_{\mathrm{F}}$ the gain of the proportional force controller and $\boldsymbol{p}=$ $\left(p_{1}, p_{2},-1, p_{4}\right)$ the tracked surface, the global control action (using force and visual information) generated by fusing force and visual information will be obtained adding the following terms (and expressing both control actions with respect to the same coordinate frame):

$$
\begin{aligned}
& \boldsymbol{v}_{\mathrm{F}}=\left[\begin{array}{c}
\mathbf{0}_{2 \times 1} \\
-\lambda_{\mathrm{F}} \cdot\left(\boldsymbol{F}-\boldsymbol{F}_{\mathrm{d}}\right)
\end{array}\right] \\
& \boldsymbol{v}_{\mathrm{I}}=-\left[\begin{array}{ll}
1 & 0 \\
0 & 1 \\
p_{1} & p_{2}
\end{array}\right]\left(\lambda_{\mathrm{v}} \cdot \widehat{\mathbf{J}}_{\mathrm{f}}^{+} \cdot\left(\mathbf{s}-\mathbf{s}_{d}(t)\right)-\widehat{\mathbf{J}}_{\mathrm{f}}^{+} \cdot \frac{\partial \mathbf{s}_{\mathrm{d}}(t)}{\partial t}\right)
\end{aligned}
$$

\section{DETECTING SURFACE DISCONTINUITIES FROM INTERACTION FORCES}

In our previous studies [15] we have shown a method to detect discontinuities on contact surfaces based on the GLR (Generalized Likelihood Ratio) [18]. This method has several possible problems and a more extensive study is shown in this paper. In previous studies, the GLR has been applied in other environments such as the detection of discontinuities of road curvatures [2]. In our case, the interaction forces are filtered by using a Kalman filter and from these filtered forces, the GLR is obtained. The value of the GLR increases when abrupt changes are determined from the obtained interaction forces, therefore, the greater the GLR the greater the probability of a change in the surface. We have defined the threshold $U$ so that if GLR $>U$, then a change in the surface is produced (empirically, this threshold has been established at 1000).

In Fig. 1, the values of the force $f_{\mathrm{z}}$ and the GLR obtained during the tracking of a curved surface, is shown. This method is not enough robust to detect the exact point in which a discontinuity in the surface occurs. For example, in Fig. 1 there are three different moments in which the discontinuity is detected (GLR is greater than 1000). As it is shown in this figure, false detections can be obtained when high frictions are generated. Furthermore, these false detections can be obtained in other situations due to the high noise level in the measured signal from the force sensor. Therefore, we conclude that this method is only adequate to obtain a value about the probability of a discontinuity in the surface. However, to improve the robustness in the detection, some additional information must be applied.

In the next section, a method based on structured light is defined in order to be combined with the one proposed in this section. The aim is to obtain a robust method for the detection of discontinuities in contact surfaces.

\section{STRUCTURED LIGHT TO DETECT DISCONTINUITIES ZONES IN THE IMAGE SPACE}

In this section a new approach to be used jointly with the previous one to detect discontinuity zones in surfaces is proposed. This approach is based on the use of patterns of structured light which are projected on the surfaces of the scene objects. The light beam projection over the different surfaces determines certain discontinuities due to the change of direction of the projection plane. The discontinuity is caused by the breakage of light beam projection; thus, several projection planes displaced in the space can be observed. The system employed for the detection process is composed by a CCD camera, a laser LASIRIS SNF with a wavelength of $660 \mathrm{~nm}$, a power of $20 \mathrm{~mW}$, a fan angle about $15^{\circ}$ and 7 concentric circles with an interbeam angle of $0.77^{\circ}$. This laser is located next to the camera in the robot end-effector. This way, the camera-laser system scans the scene in each one of the trajectories executed by the robot.

$f_{\mathrm{z}}(\mathrm{N})$

Iterations

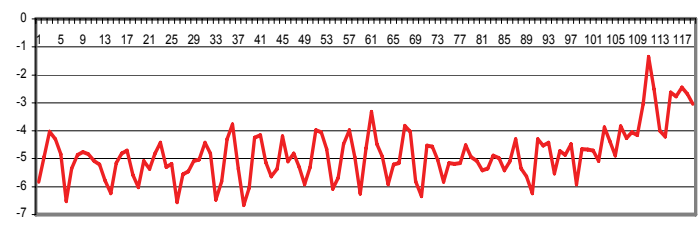

GLR

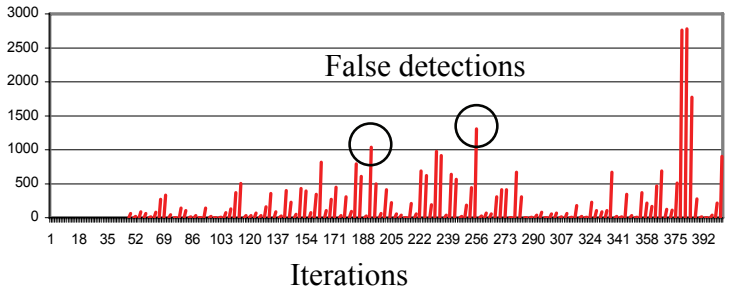

Fig. 1. Evolution of $f_{\mathrm{z}}$. and value of GLR during the tracking.

\section{A Step 1. Approximation of beam contours}

In this section, the proposed process for detecting discontinuities using structured light laser is presented [7]. Once the beam is projected over the objects surface, the first stage consists of extracting the contours of circle pattern. To 
do so, firstly, a Gaussian smoothed process with a $3 \times 3$ size mask is applied to reduce the produced noise when the beam hits the reflecting surfaces.

Later, the image is binarized by means of a suitable threshold, according to the wavelength of the laser. Experimentally, a luminance threshold about 70 has been considered for $660 \mathrm{~nm}$. With this threshold it is possible to extract only the information of contours from the projected laser pattern (Fig. 2). Next, the projected circles in the image are detected by means of a convolution process. In this process, 8-connectivity masks are used [8]. Thus, the pixels which determine each contour are found. This set of pixels corresponds to the 2D-points which compose each projected circle. Afterwards, it is necessary to approximate every contour using some kind of geometric primitive which defines the contours by means of several parameters. These parameters will be employed below in order to differentiate contours. Thus, each circular contour is represented by a polygonal approach (Fig. 2).

The advantages of a polygonal approach are two. On the one hand, to obtain a simple representation for each contour which permits a fast and efficient comparison process among contours. On the other hand, another advantage is the reduction of the number of control points detected during the contour extraction process. Thus, the beam projection is only represented by a minimum set of control points without loss of stability in the contour extraction process.

Our approach method is based on the Douglas-Peucker's algorithm [6][9]. Each contour is approximated by a poly-line, $P_{s}$. If a contour is defined as a sequence of $n$ points so that $C_{s}=\left\{p_{1}, p_{2}, \ldots, p_{n}\right\}$, a poly-line $P_{s}$, can be defined as the union of 1-degree segments, where $l$ denotes each segment.

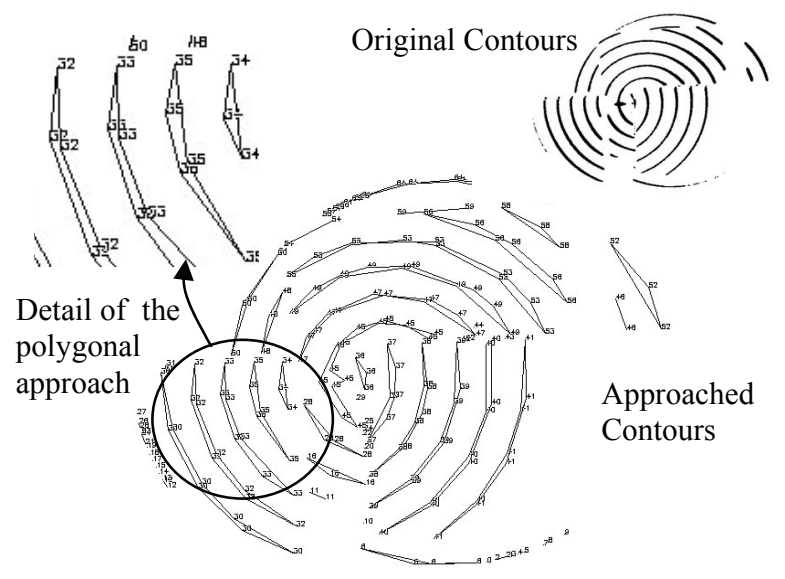

Fig. 2. Projection of the beam. Approximation of the beam

$$
\begin{aligned}
P_{s}=\bigcup_{i=1}^{n-1}\left\{l_{i} l_{i+1}\right\} & =\bigcup_{i=1}^{n-1}\left\{t p_{i}+(1-t) p_{i+1} / 0 \leq t \leq 1\right\} \\
P_{s} & =\left\{p_{1} \ldots p_{n} / n \geq 2\right\}
\end{aligned}
$$

The adjustment is based on the representation of a set of points by means of edge segments where the proximity of each point to the edge segment must be inferior to a tolerance factor $\varepsilon$ which is taken like the reference threshold. The proximity is measured like a normal distance vector to each candidate edge segment.

The polygonal adjustment process allows the approximation of the laser beam contours in a more stable way than a set of contour points. Now, each edge contour is composed by fewer points. Each contour is a set of points greater than two, which it is the minimum number of points necessary to define a segment.

Nevertheless, in spite of the previous smoothing preprocessing, during the contour detection process, some contours have been approximated by poly-lines formed by a small number of points. This fact can be due to noise, that is, contour points which contribute with little information. This is due to the fact that it represents very small and despicable discontinuities. Therefore, the approximated contours should be filtered in order to work only with those that have some length and are determined by a number of points greater than three $P_{s}=\left\{p_{1} p_{2} \ldots p_{n} / n \geq 3\right\}$.

This method does not need compute the center of laser strip because the points which determine a contour are approximated by polygonal lines and not by curves.

\section{B Step 2. Clustering and discontinuity directions}

Once the polygonal approaches have been filtered, only the end points are chosen. These points are the interest points which will determine the directions of discontinuity $P_{s}=\left\{p_{1} p_{n} / n \geq 3\right\}$.

Later, a method for the adjustment of the interest points is applied by means of straight lines. These straight lines determine the candidate zones and the suitable direction for the search of possible discontinuities which are present in the changes of surface.

A clustering process of interest points is used to determine whichever straight lines are necessary to fit them. As well to discriminate what interest points belong to one or another direction of discontinuity. The clustering process permits to group the control points according to two parameters: inertial moments [16] and metric distances [4]. Thus, each edge contour approximated by a poly-line $P_{s}$ has two interest points, each one must belong to different clusters, and must have associated the central moments of the poly-line to which they belong.

$$
\mu_{p, q}=\iint\left(x-x_{c}\right)^{p}\left(y-y_{c}\right)^{q} f(x, y) d x d y
$$

Furthermore, it is possible to determine the rotation of each edge contour around its gravity center from the central moments and with the aid of the components of inertial tensor, as: 


$$
\theta=\frac{1}{2} \arctan \frac{2 \mu_{1,1}}{\mu_{2,0}-\mu_{0,2}}
$$

The parameter of inertial moments is employed to discern between interest points which belong to poly-lines, and therefore to contours with the same orientation. This way, we have supposed that the beam projections over the same surface of an object $A$ have similar inertial moments. Although, in an object $B$ which is overlapping and occluding part of surface of $A$, the inertial moments estimated from the beam projections over a surface of $B$, are different to those estimated from $A$ (Fig. 3). When a discontinuity is detected, an orientation change of the beam projections takes places, and consequently a new value of the inertial moments is obtained.

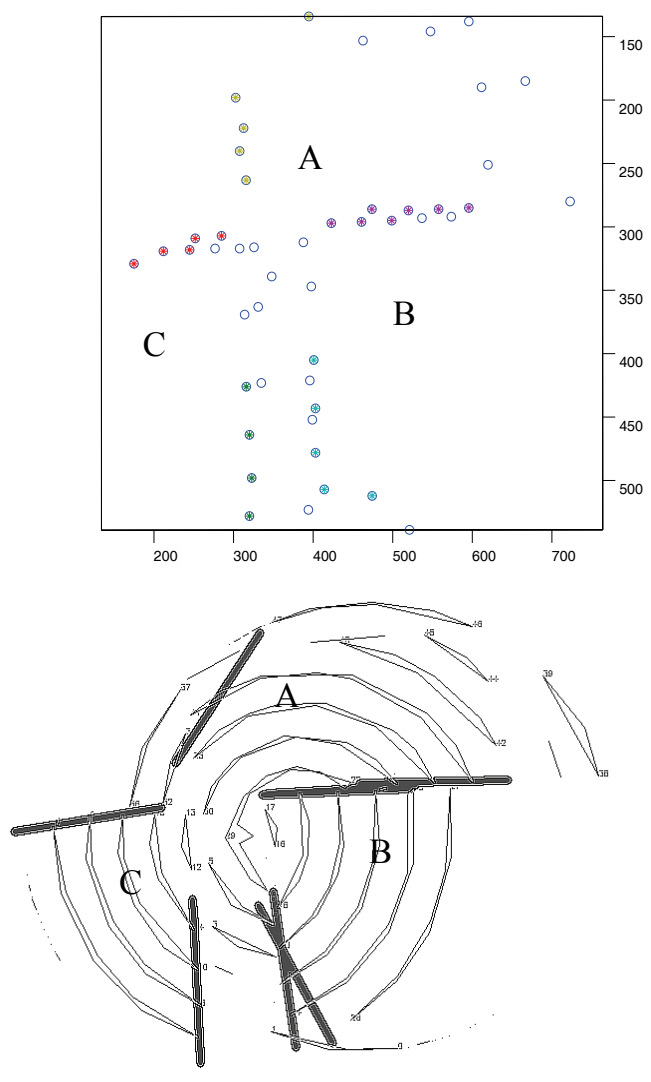

a)

Fig. 3. a) Clustering process. b) Direction of discontinuities

Also, another parameter to emphasize is the distance of Minkowski $\mathrm{L}_{2}$. Thus, the distance of each interest point to the rest of interest points is calculated, trying to minimize it.

$$
d_{L_{r}}=\left(\sum_{i, j}\left|p_{i}-p_{j}\right|^{r}\right)^{1 / r}
$$

The distance parameter is used to avoid the grouping as points of a same discontinuity, those points which can agree at inertial moment, but due to their little proximity they may not belong to the same candidate zone. For this reason, only the interest points, near among them and whose poly-lines have similar inertial moments, are clusterized.

Then, we obtain $n$ sets of points of the following type: $p_{i}=\left\{s_{1} s_{2} \ldots s_{n}\right\} / 0<i<n$ where $\left\{s_{1} s_{2} \ldots s_{n}\right\}$ are the points nearer $p_{i}$ ordered according to proximity. Finally, the difference of moments for each set of points is obtained as it was mentioned above.

$$
d \theta_{i j}=\left|\theta_{p i}-\theta_{s j}\right|
$$

From the distances and the difference of moments calculated for each set $p_{i}=\left\{s_{1} s_{2} \ldots s_{n}\right\}$, the clustering process is made (Fig. 3.a). The candidate zones which represent discontinuities in the image are defined as those zones which contain each cluster. In order to determine the search direction of those candidate zones and their boundary, it is necessary to make a linear adjustment by least-squares method which calculates the straight line segments which diminish the Minkowski's distance $\mathrm{L}_{1}-\mathrm{L}_{2}$ of each set of interest points to the straight line segment (Fig. 3.b).

\section{ROBUST MULTISENSORIAL SYSTEM TO DETECT CHANGES IN SURFACES}

When the system described in Section IV based on structured light determines a discontinuity zone, a line is obtained in the image space which represents this discontinuity ${ }^{0} \mathbf{l}_{\mathrm{d}}$. As it is shown in Fig. 4, in the image it is possible to determine not only the visual features employed by the visual servoing system $\left({ }^{\mathrm{k}} \boldsymbol{f}_{1}{ }^{\mathrm{k}} \boldsymbol{f}_{2}{ }^{\mathrm{k}} \boldsymbol{f}_{3}{ }^{\mathrm{k}} \boldsymbol{f}_{4}\right)$, but also the point in the image, $\mathbf{p}_{\mathrm{c}}$, which corresponds to the contact between the end-effector tool and the surface. Therefore, the algorithm described in Section III will be activated at the moment in which $\mathbf{p}_{\mathrm{c}}$ arrives to the discontinuity line ${ }^{0} \mathbf{l}_{\mathrm{d}}$, in this way avoiding false detections. The estimation of the iteration at which the point $\mathbf{p}_{\mathrm{c}}$ is near the straight line ${ }^{0} \mathbf{l}_{\mathrm{d}}$ is the object of this section.

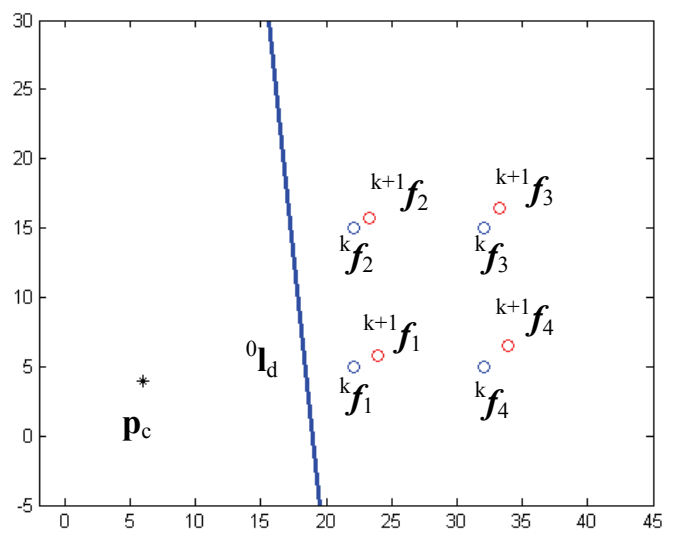

Fig. 4. Features extracted in the image and discontinuity line ${ }^{0} \mathbf{l}_{\mathrm{d}}$.

Once the line ${ }^{0} \mathbf{l}_{d}$ is detected, its position in future iterations is estimated using information about the position of the 
features in the image employed by the visual servoing system. In a certain iteration, the current position of the features ${ }^{\mathrm{k}} \mathbf{s}$, is known, and the position of the desired features, ${ }^{\mathrm{k}+1} \mathbf{s}$, is obtained from the function $\mathbf{s}_{\mathrm{d}}(\mathrm{t})$ described in Section II. Using this information it can be calculated the homography matrix relating these two positions $\mathbf{G}_{\mathrm{k}}$ as it is described in [8]. Fig. 4 shows the current and the desired position of the visual features at a certain iteration of the visual servoing task.

Once the homography matrix is calculated, it is possible to obtain the rotation $\mathbf{R}_{\mathrm{k}}$ and the translation $\mathbf{t}_{\mathrm{k}}$ that the camera has undergone from $\mathbf{G}_{\mathrm{k}}$, applying the algorithms shown in [19]. Once the camera rotation and translation has been obtained, it is possible to locate in the following iteration,${ }_{1} \mathbf{l}_{d}$, the straight line ${ }^{0} \mathbf{l}_{d}$ given by the algorithm described in section IV. To do so, two points located on the line are considered. Applying (10), the position of each point is determined from the camera motion previously determined:

$$
\boldsymbol{x}_{1}=\mathbf{R}_{\mathrm{k}}^{-1}\left(\boldsymbol{x}_{0}-\mathbf{t}_{\mathrm{k}}\right)
$$

where $\boldsymbol{x}_{0}$ and $\boldsymbol{x}_{1}$ are the coordinates of the point in the image at the current iteration and at the position obtained after the camera motion. By calculating the motion of the two points, the position of the virtual line ${ }^{\mathrm{i}} \mathbf{l}_{\mathrm{d}}$ in the image plane can be reconstructed.

The algorithm computes the position of the straight line ${ }^{\mathrm{i}} \mathbf{I}_{d}$ at each iteration of the visual control task until the end-effector of the robot, $\mathbf{p}_{\mathrm{c}}$, is near this line. At this moment it is employed the algorithm described in Section III to determine with precision the exact point in which the discontinuity occurs. In Fig. 5 it can be seen the simulation of the position of the virtual line ${ }^{0} \mathbf{I}_{d}$ during the visual servoing task. The endeffector, $\mathbf{p}_{\mathrm{c}}$, has a fixed position in the image plane (there is a constant distance between the camera and the end-effector). The initial position is indicated by ${ }^{0} \mathbf{l}_{d}$. The lines represented as ${ }^{\mathrm{i}} \mathbf{l}_{\mathrm{d}}$ are the successive estimations obtained by the algorithm described in this section.

Finally, the line ${ }^{f} \mathbf{l}_{\mathrm{d}}$ represents the obtained position at the iteration in which the algorithm detects that the tool at the endeffector is satisfactorily close to this line. At this point, the method described in Section III is activated.

\section{RESULTS}

\section{A System architecture}

The system architecture is composed of an eye-in-hand PHOTONFOCUS MV-D752-160-CL-8 camera at the endeffector of a 7 d.o.f. Mitsubishi PA-10 robot (see Fig. 6) also equipped with a force sensor and a laser LASIRIS SNF with a wavelength of $660 \mathrm{~nm}$. The camera is able to acquire and to process up to 100 frames/second using an image resolution of $320 \times 240$. In this paper we are not interested in image processing issues, therefore, the image trajectory is generated by using four grey marks whose centres of gravity will be the extracted features.

Now, an experiment in which the robot tracks a surface with a discontinuity is described. The discontinuity of the surface is shown in Fig. 7. The interaction forces and the value of GLR obtained in the tracking of the surface using the approximation described in Section III are shown in Fig. 8. We can observe that the system is able to detect the discontinuity zone, but also provides false detections. To improve the system behaviour the next step is to limit the zones where the discontinuity can appear by using the method based on structured light described in Section IV.

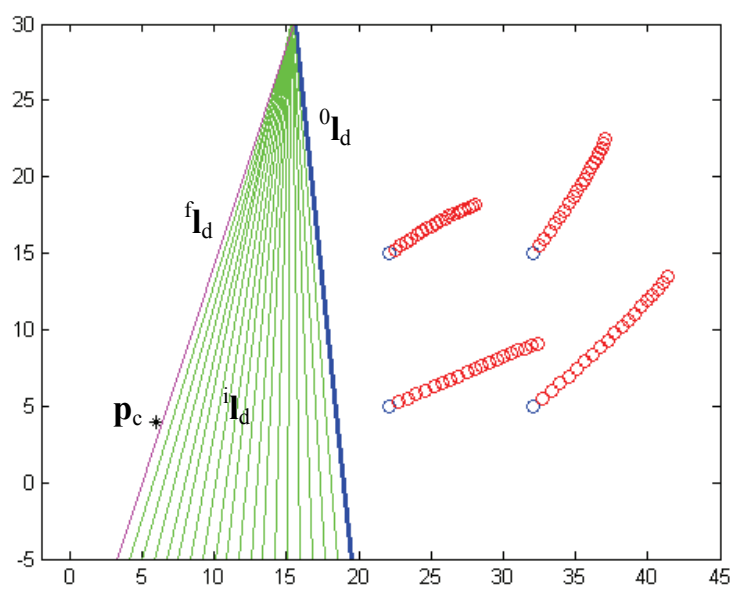

Fig. 5. Projection of the discontinuity during the visual servoing task.

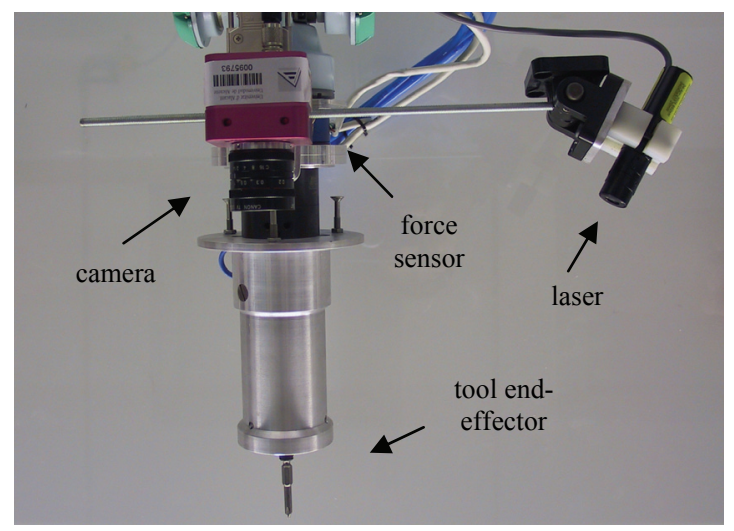

Fig. 6. Experimental setup.

Using this method, the discontinuity zone represented by two straight lines in Fig. 9.a is obtained. The last step is to determine when the end-effector is located near the discontinuity zone. This way the algorithm described in Section III is only executed in this zone, avoiding false detections. Following the process described in Section V, once 
the discontinuity line is detected ${ }^{0} \mathbf{l}_{\mathrm{d}}$, the rotation and translation of the camera is determined at each iteration of the visual servoing task. This information is used to project the position of the discontinuity line at each iteration, ${ }^{i} \mathbf{I}_{\mathrm{d}}$. Therefore when this line is sufficiently near to the position of the end-effector, the algorithm described in Section III is executed to determine the exact point of the discontinuity (this process is illustrated in Fig. 9.b).

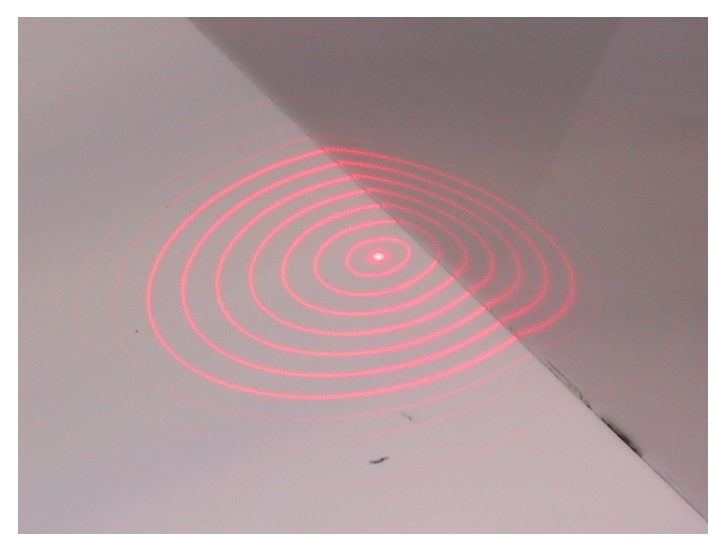

Fig. 7. Surface discontinuity.
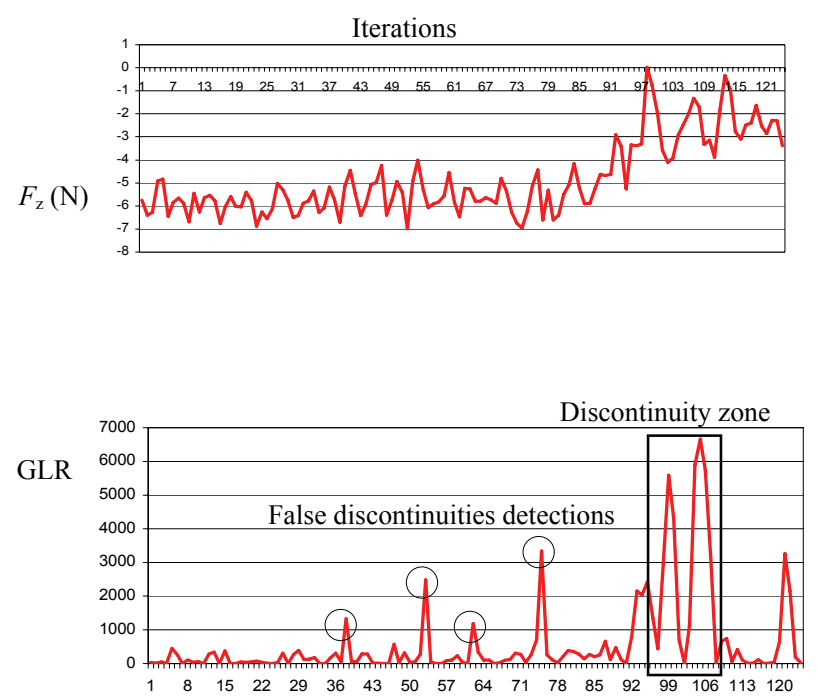

Iterations

Fig. 8. Evolution of $f_{\mathrm{z}}$. and value of GLR during the tracking.

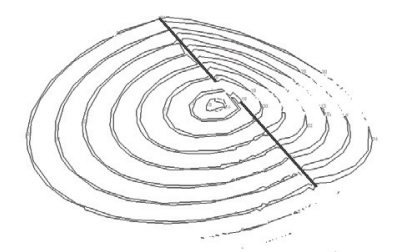

a)

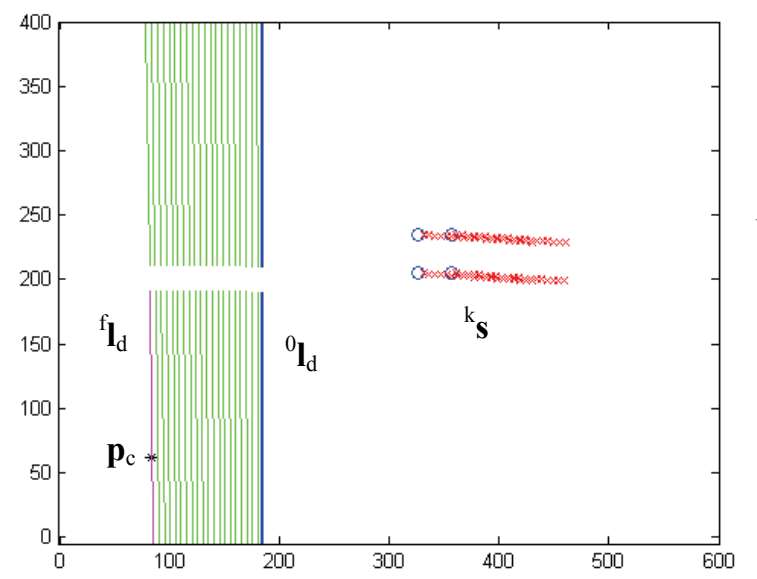

Fig. 9. a) Zone of discontinuity using the method described in Section IV. b) Process carried out to determine the point in which the end-effector arrives to the discontinuity.

\section{CONCLUSIONS}

In this paper, a method to detect discontinuities in surfaces using a visual-force control tasks is described. To do so, first an approach to detect these discontinuities by only using force information has been shown. However, these approaches are sensitive to frictions as well as the high noise level of the force sensor signal. To improve the detections we have included additional sensorial information from a laser. Using structured light, the system is able to automatically detect zones where the discontinuity can occur. They are only considered correct when the discontinuities that are determined by the force sensor are near the determined structured light. This method has been proved to be robust even in situations in which the surface has soft discontinuities.

Presently, we are working in improving the surface recognition method. This method modifies the image trajectory depending on the information obtained from the force sensor.

\section{ACKNOWLEDGMENT}

This work was funded by the Spanish MCYT project "Diseño, implementación y experimentación de escenarios de manipulación inteligentes para aplicaciones de ensamblado y desensamblado automático" and by the project GV05/007: 
"Diseño y experimentación de estrategias de control visualfuerza para sistemas flexibles de manipulación".

\section{REFERENCES}

[1] J. Baeten, J. De Schutter, "Hybrid Vision/Force Control at Corners in Planar Robotic-Contour Following" IEEE/ASME Transactions on Mechatronics, vol. 7, no 2, pp. 143 - 151. 2002.

[2] R. Behringer, "Detection of discontinuities of road curvature by glr methods". Proceedings of International Symposium on Intelligent Vehicles. Detroit, USA. 1995.

[3] H. Bruyninckx, J. De Schutter, "Specification of forcecontrolled actions in the task frame formalism-A synthesis", IEEE Transactions on Robotics and Automation, vol. 12, no. 4. pp. 581-589. 1996.

[4] E. Chavez, G. Navarro, R. Baeza-Yates, J. Marroquín, "Searching in metric spaces" Technical Report TR/DCC-99-3, Dept. of Computer Science, Univ. of Chile. 1999.

[5] De Schutter, J.; Bruyninckx, H.; Spong, M. "Force control : a bird's eye view". in IEEE International Workshop on Control Problems in Robotics and Automation : Future Directions. San Diego, CA, 1997.

[6] D. Douglas, T. Peucker, "Algorithms for the reduction of the number points required to represent a digitized line or its caricature". The Canadian Cartographer, vol. 10, no. 2, pp. 112-122. 1973.

[7] P. Gil, F. Torres, O. Reinoso, "A detection method of intersections for determining overlapping using active vision". International Conference on Computer Vision Theory and Applications, VISAPP 2006, vol. 1, pp. 501-507, Setubal, Portugal, 2006.

[8] R. Hartley, A. Zisserman. "Multiple view Geometry in Computer Vision". Cambridge University Press, pp. 91 - 92. 2000.

[9] J. Hershberger, J. Snoeyink, "Speeding Up the Douglas-Peucker Line-Simplification Algorithm". Proc. $5^{\text {th }}$ Symp. On Data
Handling, pp. 134-143. 1993.

[10] S. Hutchinson, G. D. Hager, and P. I. Corke, "A tutorial on visual servo control”. IEEE Trans. Robot. Automat., vol. 12, no. 5, pp. 651-670, Oct. 1996.

[11] A. Krupa, J. Gangloff, C. Doignon, M. Mathelin, G. Morel, J. Leroy, L. Soler, and J. Marescaux, "Autonomous 3d positionning of surgical instruments in robotized laparoscopic surgery using visual servoing".IEEE Trans. on Robotics and Automation, vol. 19, no. 5, pp. 842-853. 2003.

[12] Y. Mezouar and F. Chaumette, "Path Planning For Robust Image-based Control", IEEE Trans. on Robot. Automat., vol. 18, no. 4, pp. 534-549, Aug. 2002.

[13] J. Pagès, C. Collewet, F. Chaumette and J. Salvi. "Robust decoupled visual servoing based on structured light". IEEE/RSJ International Conference on Intelligent Robots and Systems, IROS 2005, pp. 2676-2681, Edmonton, Canada. 2005.

[14] J. Pomares, S. T. Puente, F. Torres, F. A. Candelas, P. Gil. "Virtual disassembly of products based on geometric models", Jounal of Computers in Industry. Vol 55, no. 1. pp. 1-14. 2004.

[15] J. Pomares, F. Torres, "Movement-flow based visual servoing and force control fusion for manipulation tasks in unstructured environments". IEEE Transactions on Systems, Man, and Cybernetics-Part C. Vol. 35, No. 1. Pp. 4-15. 2005.

[16] M. R. Teague, "Image Análisis via the general theory of moments". Journal of Optical Society of America 70(8), pp. 920-930. 1980.

[17] C. H. Teh, R. T. Chin, "On the detection of dominant points on digital curves" IEEE Trans. PAMI 1(8), pp. 859-872. 1989.

[18] A. S. Willsky, H. L. Jones. "A generalized likelihood ration approach to the detection and estimation of jumps in linear systems". IEEE Trans. Automat. Contr., vol. 21, no. 1, pp. 108-112. 1976.

[19] Z. Zhang, A. R. Hanson, "Scaled Euclidean 3D reconstruction based on externally uncalibrated cameras", Proc IEEE International Conference on Robotics and Automation, San Francisco, CA., 1995. 